\title{
Thermal Characteristics of Vertical Buoyant Jet from a Square Orifice in
}

\section{Static Ambient}

\author{
Wang Shaobo ${ }^{1, a}$, Cui Liqin ${ }^{1, b}$ and Sun Xiaoli, ${ }^{2, c}$ \\ ${ }^{1}$ Pearl River Hydraulic Research Institute, Guangzhou 510611, China \\ ${ }^{2}$ Pearl River Water Resources Commission of the Ministry of Water Resources, Guangzhou \\ 510611, China

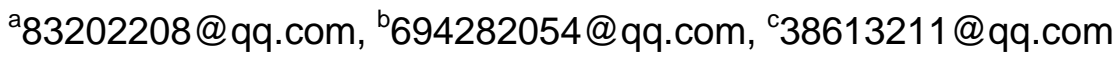

Key words: buoyant jet, square orifice, dilution, numerical simulation, static ambient, experiment Abstract:A three-dimensional eddy model considering buoyancy was established to simulate vertical buoyant flow from a square orifice in static ambient. The hybrid finite analytic method with stagged grid was used to solve this model. Experiments were made to confirm the efficiency and validity of the model, and concentration on the axis under different experimental conditions was obtained. Conclusions showed that axial concentration from a square orifice coincided well with those from a circular one after a little distance from the jet mouth. The shapes of temperature distribution on different height cross-sections tallied well with those from a circular orifice. The existence of self-similar area of temperature was confirmed also.

\section{Introduction}

Systemic review and summarization on round vertical turbulent buoyant jets have been made by List (1982) ${ }^{[1]}$ and Chen and Rodi (1980) ${ }^{[2]}$. Extensive reviews have been reported by Jirka, Abraham and Harleman (1975) ${ }^{[3]}$ as well as by Fischer et al. (1979) ${ }^{[4]}$. Unified formulas of axial concentration and velocity for jets with or not buoyancy have been proposed by Noutsopoulos and Yannopoulos (1987) $)^{[5]}$, in which integral forms of the momentum and tracer equations were integrated on the basis of an assumption concerning a function of the spreading coefficients. The dilution characteristics of obstructed buoyant vertical jets in static ambient were also studied by Huai et al (2006) ${ }^{[6]}$ and Huai and Fang (2006) ${ }^{[7]}$. However, study about vertical turbulent buoyant jets from a square orifice are relatively rare. Experiments and numerical simulations have been made on vertical turbulent buoyant jets of thermal water from a square orifice in static ambient to compare with those from a round orifice.

\section{Mathematical Model}

A three-dimensional one must be used in numerical simulations of jets from a square orifice. Dimensionless turbulent RNG $k-\varepsilon$ mathematical model is as following based on characteristic quantities of exit velocity $v_{j}$, side length of the square orifice $d$ and ambient water density $\rho_{a}$ :

$$
\begin{gathered}
\frac{\partial u}{\partial x}+\frac{\partial v}{\partial y}+\frac{\partial w}{\partial z}=0 \\
\operatorname{Re}_{e t}\left(u-2 \frac{\partial v_{t}}{\partial x}\right) \frac{\partial u}{\partial x}+\operatorname{Re}_{e t}\left(v-\frac{\partial v_{t}}{\partial y}\right) \frac{\partial u}{\partial y}+\operatorname{Re}_{e t}\left(w-\frac{\partial v_{t}}{\partial z}\right) \frac{\partial u}{\partial z}= \\
\left(\frac{\partial^{2} u}{\partial x^{2}}+\frac{\partial^{2} u}{\partial y^{2}}+\frac{\partial^{2} u}{\partial z^{2}}\right)+\operatorname{Re}_{e t}\left(-\frac{\partial p}{\partial x}-\frac{2}{3} \frac{\partial k}{\partial x}+\frac{\partial v_{t}}{\partial y} \frac{\partial v}{\partial x}+\frac{\partial v_{t}}{\partial z} \frac{\partial w}{\partial x}\right)
\end{gathered}
$$




$$
\begin{aligned}
& \operatorname{Re}_{e t}\left(u-\frac{\partial v_{t}}{\partial x}\right) \frac{\partial v}{\partial x}+\operatorname{Re}_{e t}\left(v-2 \frac{\partial v_{t}}{\partial y}\right) \frac{\partial v}{\partial y}+\operatorname{Re}_{e t}\left(w-\frac{\partial v_{t}}{\partial z}\right) \frac{\partial v}{\partial z}= \\
& \left(\frac{\partial^{2} v}{\partial x^{2}}+\frac{\partial^{2} v}{\partial y^{2}}+\frac{\partial^{2} v}{\partial z^{2}}\right)+\operatorname{Re}_{e t}\left(-\frac{\partial p}{\partial y}-\frac{2}{3} \frac{\partial k}{\partial y}+\frac{\partial v_{t}}{\partial x} \frac{\partial u}{\partial y}+\frac{\partial v_{t}}{\partial z} \frac{\partial w}{\partial y}\right)+\beta g \Delta T \frac{d}{v_{j}^{2}} \\
& \operatorname{Re}_{e t}\left(u-\frac{\partial v_{t}}{\partial x}\right) \frac{\partial w}{\partial x}+\operatorname{Re}_{e t}\left(v-\frac{\partial v_{t}}{\partial y}\right) \frac{\partial w}{\partial y}+\operatorname{Re}_{e t}\left(w-2 \frac{\partial v_{t}}{\partial z}\right) \frac{\partial w}{\partial z}= \\
& \left(\frac{\partial^{2} w}{\partial x^{2}}+\frac{\partial^{2} w}{\partial y^{2}}+\frac{\partial^{2} w}{\partial z^{2}}\right)+\operatorname{Re}_{e t}\left(-\frac{\partial p}{\partial z}-\frac{2}{3} \frac{\partial k}{\partial z}+\frac{\partial v_{t}}{\partial x} \frac{\partial u}{\partial z}+\frac{\partial v_{t}}{\partial y} \frac{\partial v}{\partial z}\right) \\
& \operatorname{Re}_{e k}\left(u-\frac{1}{\sigma_{k}} \frac{\partial v_{t}}{\partial x}\right) \frac{\partial k}{\partial x}+\operatorname{Re}_{e k}\left(v-\frac{1}{\sigma_{k}} \frac{\partial v_{t}}{\partial y}\right) \frac{\partial k}{\partial y}+\operatorname{Re}_{e t}\left(w-\frac{1}{\sigma_{k}} \frac{\partial v_{t}}{\partial z}\right) \frac{\partial k}{\partial z}= \\
& \left(\frac{\partial^{2} k}{\partial x^{2}}+\frac{\partial^{2} k}{\partial y^{2}}+\frac{\partial^{2} k}{\partial z^{2}}\right)+\operatorname{Re}_{e k}\left(P_{k}-\varepsilon+\alpha g \frac{v_{t}}{P_{r t}} \frac{\partial T}{\partial z} \frac{d}{v_{j}^{2}}\right) \\
& \operatorname{Re}_{e \varepsilon}\left(u-\frac{1}{\sigma_{k}} \frac{\partial v_{t}}{\partial x}\right) \frac{\partial \varepsilon}{\partial x}+\operatorname{Re}_{e \varepsilon}\left(v-\frac{1}{\sigma_{k}} \frac{\partial v_{t}}{\partial y}\right) \frac{\partial \varepsilon}{\partial y}+\operatorname{Re}_{e \varepsilon}\left(w-\frac{1}{\sigma_{k}} \frac{\partial v_{t}}{\partial z}\right) \frac{\partial \varepsilon}{\partial z}= \\
& \left(\frac{\partial^{2} \varepsilon}{\partial x^{2}}+\frac{\partial^{2} \varepsilon}{\partial y^{2}}+\frac{\partial^{2} \varepsilon}{\partial z^{2}}\right)+\operatorname{Re}_{e \varepsilon}\left(C_{\varepsilon 1} \frac{\varepsilon}{k}\left(P_{k}+\alpha g \frac{v_{t}}{P_{r t}} \frac{\partial T}{\partial y} \frac{d}{v_{j}^{2}}\right)-C_{\varepsilon 2} \frac{\varepsilon^{2}}{k}\right) \\
& \operatorname{Re}_{e T}\left(u-\frac{1}{\mathrm{P}_{r t}} \frac{\partial v_{t}}{\partial x}\right) \frac{\partial T}{\partial x}+\operatorname{Re}_{e T}\left(v-\frac{1}{\mathrm{P}_{r t}} \frac{\partial v_{t}}{\partial y}\right) \frac{\partial T}{\partial y}+\operatorname{Re}_{e t}\left(w-\frac{1}{\mathrm{P}_{r t}} \frac{\partial v_{t}}{\partial z}\right) \frac{\partial T}{\partial z}= \\
& \left(\frac{\partial^{2} T}{\partial x^{2}}+\frac{\partial^{2} T}{\partial y^{2}}+\frac{\partial^{2} T}{\partial z^{2}}\right)
\end{aligned}
$$

where $x$ and $z$ denote horizontal directional coordinate and $y$ vertical directional coordinate. $u, v$ and $w$ are velocities in $x, y$ and $z$ direction. $p, k, \varepsilon, T$ denote pressure, turbulent kinetic energy, energy dissipation rate and temperature respectively. $P_{r}$ and $P_{r t}$ are Prandtl numbers with value of 0.7 used here. $v$ and $v_{t}$ are kinetic viscosity coefficients. $R_{e t}, R_{e k}, R_{e \varepsilon}$ and $R_{e T}$ are dimensionless coefficients in deducing above equations and expressed as following:

$$
R_{e t}=\frac{\operatorname{Re}}{1+\operatorname{Rev}_{t}}, \quad R_{e k}=\frac{\operatorname{Re}}{1+\operatorname{Rev}_{t} / \sigma_{k}}, \quad R_{e \varepsilon}=\frac{\operatorname{Re}}{1+\operatorname{Rev}_{t} / \sigma_{\varepsilon}}, \quad R_{e T}=\frac{1}{1 /\left(\operatorname{Re} P_{r}\right)+v_{t} / P_{r t}}
$$

where Re is Reynolds number with $\operatorname{Re}=\frac{v_{j} d}{v}$ and $v_{t}=C_{\mu} \frac{k^{2}}{\varepsilon}$. In equation (5) and (6):

$$
P_{k}=v_{t}\left[2\left(\frac{\partial u}{\partial x}\right)^{2}+2\left(\frac{\partial v}{\partial y}\right)^{2}+2\left(\frac{\partial w}{\partial z}\right)^{2}+\left(\frac{\partial v}{\partial x}+\frac{\partial u}{\partial y}\right)^{2}+\left(\frac{\partial v}{\partial z}+\frac{\partial w}{\partial y}\right)^{2}+\left(\frac{\partial w}{\partial x}+\frac{\partial u}{\partial z}\right)^{2}\right]
$$

Constants in the model are as following:

$C_{\mu}=0.085, \quad C_{\varepsilon 1}=1.42-\frac{\eta\left(1-\eta / \eta_{0}\right)}{1+\beta \eta^{3}}, \quad \eta=S k / \varepsilon, S=\left(P_{k} / \nu_{t}\right)^{1 / 2}, \quad \beta=0.015, C_{\varepsilon 2}=1.68, \quad \sigma_{\mathrm{k}}=0.7179, \quad \sigma_{\varepsilon}=0.7179$

$\Delta T=(T-1) t_{a}$ is extra temperature and $t_{a}$ is local environmental temperature. $\beta$ is thermal coefficient of expansion of water and a function for it proposed by Batchelor (1970) ${ }^{[8]}$ as following:

$$
\beta(t)=\left(-0.773+0.19 t-0.0027 t^{2}+0.000021 t^{3}\right) \times 10^{-4}
$$

in which $t$ is local temperature with its unit of degree Celsius. 


\section{Boundary Conditions}

A vertical buoyant jet from a square orifice located on a bed in semi-infinite static ambient was simulated and boundary conditions for six boundary surfaces with exit temperature $t_{j}$ as following:

Bottom boundary with $y=0$ :

$u=0, v=1, w=0, \quad T=t_{j} / t_{a}, \quad k_{0}=0.006, \quad \varepsilon_{0}=0.0001$ at the exit of the orifice;

The wall functions was used to relate the values of $\mathrm{u} 、 \mathrm{w} 、 \mathrm{k}, \varepsilon$ at the first grid points outside the viscous sub layers to boundary conditions with $v=0$ and heat insulation was applied with $a T / a y=0$ out of the exit.

Left and right boundary:

$$
\frac{\partial u}{\partial x}=\frac{\partial v}{\partial x}=\frac{\partial w}{\partial x}=\frac{\partial k}{\partial x}=\frac{\partial \varepsilon}{\partial x}=\frac{\partial T}{\partial x}=0
$$

Fore and after boundary:

$$
\frac{\partial u}{\partial x}=\frac{\partial v}{\partial x}=\frac{\partial w}{\partial x}=\frac{\partial k}{\partial x}=\frac{\partial \varepsilon}{\partial x}=\frac{\partial T}{\partial x}=0
$$

Upper boundary:

$$
\frac{\partial u}{\partial y}=\frac{\partial v}{\partial y}=\frac{\partial w}{\partial y}=\frac{\partial k}{\partial y}=\frac{\partial \varepsilon}{\partial y}=\frac{\partial T}{\partial y}=0
$$

\section{Method of Calculation}

The uniform format deduced from above partial differential equations (2) (7) in the three dimensional mathematical model can be written as following:

$$
\frac{\partial^{2} \Phi}{\partial x^{2}}+\frac{\partial^{2} \Phi}{\partial y^{2}}+\frac{\partial^{2} \Phi}{\partial z^{2}}=2 A \frac{\partial \Phi}{\partial x}+2 B \frac{\partial \Phi}{\partial y}+2 C \frac{\partial \Phi}{\partial z}+G
$$

Hybrid finite analytic method was used to solve the equation (13). The stagger grid was applied to avoid improper sawtooth-like pressure distribution arisen probably from calculating all the variables on a same node. Non-uniform grid distribution was employed for calculation. The size of calculated domain and number of grid would be adjusted lightly according to different conditions. Convergence is declared when the normalized residual is less than $1 \times 10^{-3}$ for equations of $u, v, w$ and $1 \times 10^{-5}$ for others.

\section{Experiments}

Experiments were performed in a tank made of iron sheets of rectangular plan section $150 \mathrm{~cm} \times 80$ $\mathrm{cm}$ and a depth of $80 \mathrm{~cm}$. Figure 1 shows the sketch of the whole experimental equipments. The left and right walls were formed as sharp crested weirs to keep the water surface practically constant. A small flume allocated for each side collected the overflow. The fore-and-aft walls were made of 1 $\mathrm{cm}$-thick glass for observational purposes. A $30 \mathrm{~cm}$-long aluminum pipe with a square cross section and the length of each side $l=1 \mathrm{~cm}$, was placed vertically through the center of the tank bottom, protruding $1 \mathrm{~cm}$ into the tank. 


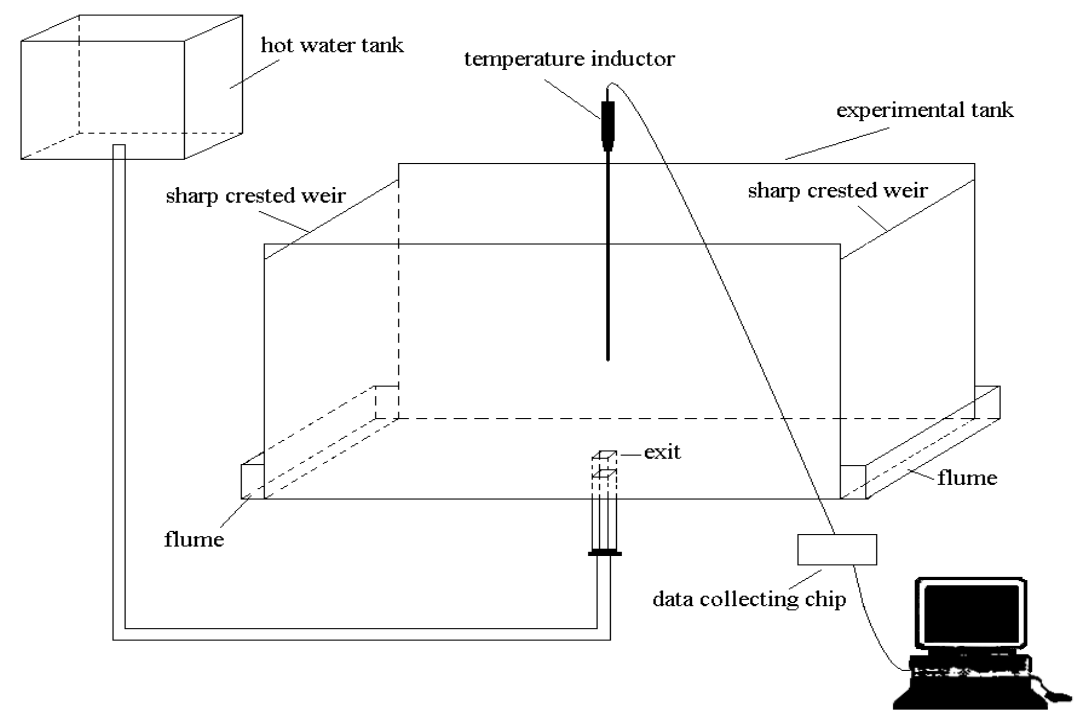

Fig.1 Sketch of experimental equipment

The system of temperature measuring is composed of two parts: data collection and data processing. Data collection includes temperature inductor and collecting chip. The inductor can be fabricated as any shape according to different requests. The local temperature detected by the inductor was transformed to collecting chip through transmission lines. The precision is $0.1^{\circ} \mathrm{C}$ and time extension used to calculate average temperature can be adjusted freely $(20$ seconds used in these experiments).

\section{Axial Concentration in Self-Similar Area}

For the convenience of comparison, local temperature obtained by calculation was transformed to local density based on corresponding relation of temperature and density for water. Effective gravity acceleration is defined as $g^{\prime}=g \Delta \rho_{0} / \rho_{a}$, in which $\Delta \rho_{0}=\rho_{a}-\rho_{j}$, g is acceleration due to gravity, $\rho_{a}$ is the density of ambient liquid, slightly larger than exit density $\rho_{j}$. Density Froude number is defined as $F_{0}=v / \sqrt{g^{\prime} d}, d$ is the side length of the square orifice. Axial concentration is denoted by $c_{m}=\left(\rho_{a}-\rho_{m}\right) /\left(\rho_{a}-\rho_{j}\right)$, where $\rho_{m}$ is density along the jet axis. Experiments had been made under density Froude number of 10.69 and 21.4 and axial temperature measured. Following Chen and Rodi (1976) ${ }^{[9]}$ and introducing the same scaling law, that is for the length:

$$
Y=(y / d) F_{0}^{-1}
$$

and for the concentration:

$$
S=c_{m} F_{0}
$$

Axial concentration of buoyant jets from a square orifice by experiments and numerical simulations were shown in Fig.2 based on the scaling laws (14) and (15), while those calculated by formulations proposed by Noutsopoulos and Yannopoulos (1987) and Chen and Rodi (1976) for buoyant jets from a round orifice also displayed. It indicates clearly that axial concentration of jets from a square orifice follows well with those from a round one. 


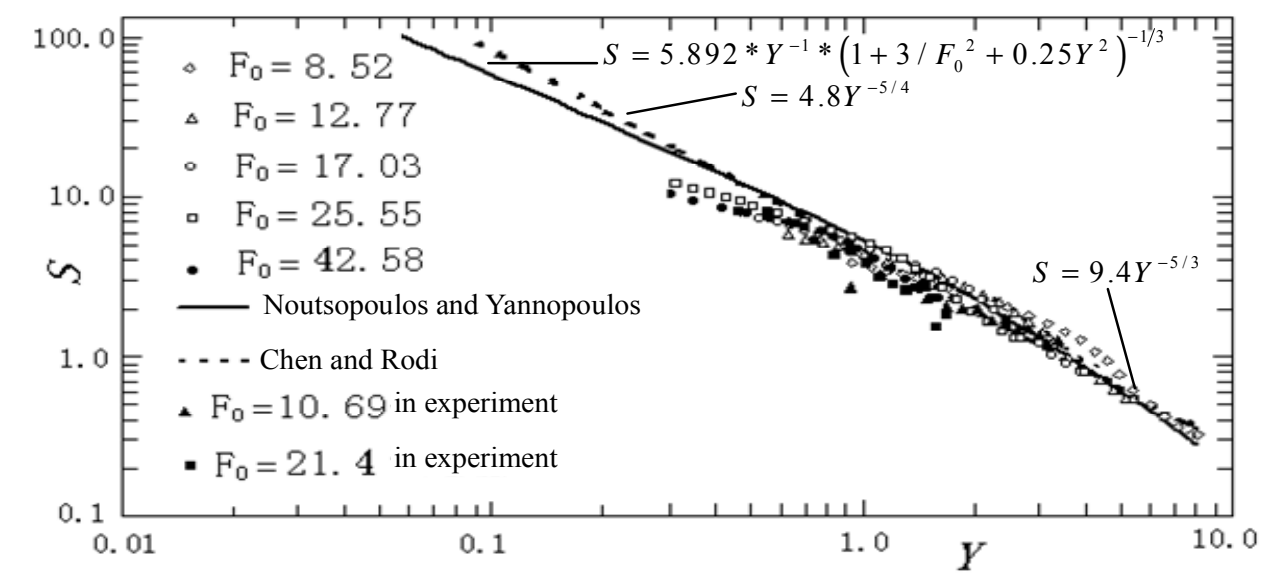

Fig.2 Comparison of axial concentration of buoyant jets from a square and round orifice

\section{Temperature by Numerical Simulations}

Contours of temperature on cross sections with different height under Froude number $F_{0}=12.77$ were shown in Fig.3 by numerical simulations. The shapes of contours change quickly from polygon $(y / d=0.5)$ to concentric circle after a distance $(y / d=1.25)$ and keep it all along, similar to those of buoyant jets from a round orifice. Temperature intersecting with the vertical axes on a cross section with different heights after a distance $(y / d=1.25)$ was displayed in Fig.4 $\left(F_{0}=8.52\right)$. It illuminates existence of the characteristic of self-similarity for horizontal temperature or concentration on a cross section with various heights, also similar to those of buoyant jets from a round orifice. The region, affected by temperature or concentration on a horizontal section, was limited to $-0.18 \leq x / y \leq 0.18$, irrespective of Froude number.
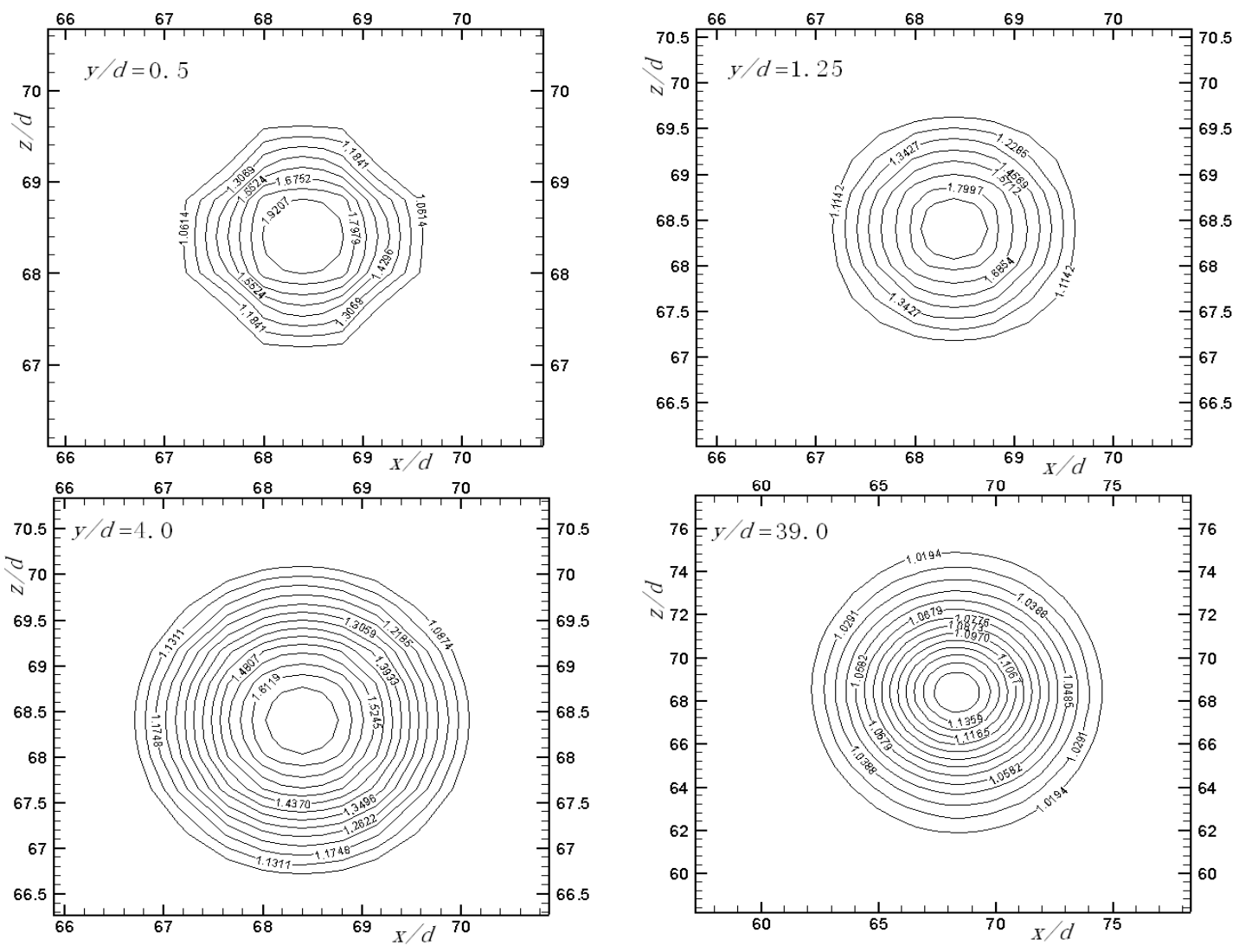

Fig.3 Temperature contours on different heights for $F_{0}=12.77$ 


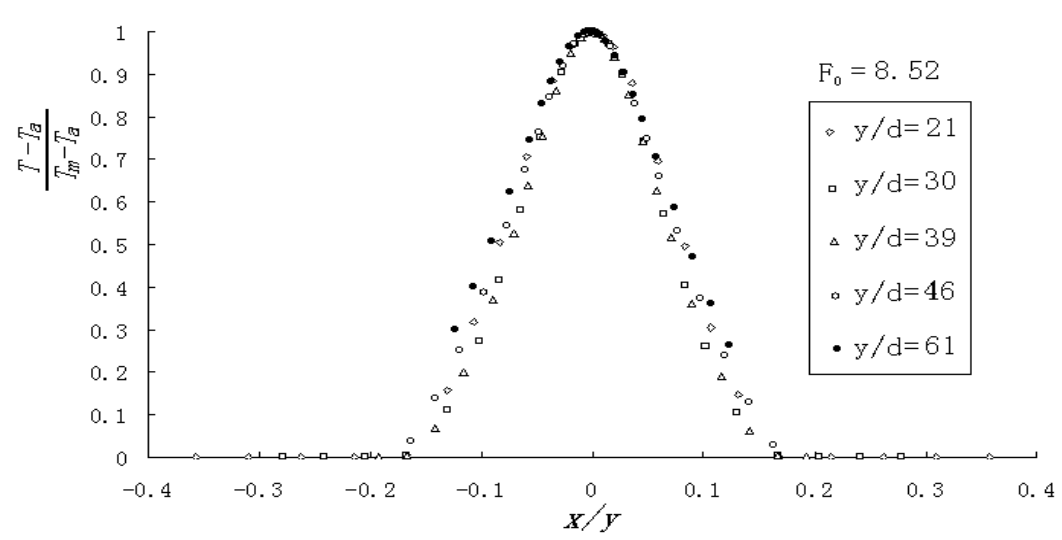

Fig. 4 Temperature distribution on different cross sections for $\mathrm{F} 0=8.52$

\section{Conclusions}

Thermal water buoyant jet from a square orifice in a semi-infinite static ambient was studied by using the methods of three-dimensional mathematical model and experiments and axial concentration under various density Froude number have been gained. Efficiency of the mathematical model was validated by well coincidence of results from numerical simulations and experiments. It shows that axial concentration from a square orifice can be predicted by the formulas of a round vertical buoyant jet. The characteristic of self-similarity of concentration on a cross section with various heights after a short distance from exit has also been validated for vertical buoyant jet from a square orifice, which is also completely similar to those from a round one.

\section{References}

[1]List E J. 1982. Mechanics of turbulent buoyant jets and plumes: In Turbulent Buoyant Jets and Plumes, Pergamon, pp: 1-68.

[2]Chen C J, Rodi W. 1980. Vertical turbulent buoyant jets: A review of experimental data, HMT, Series 4, Pergamon Press, Oxford.

[3]Jirka G H, Abraham G, Harleman D R F. 1975. An assessment of techniques for hydrothermal prediction, Report No.203. M.I.T., Ralph M. Parsons Lab.

[4]Fischer H B, List E J, Imberger J, Brooks N H. 1979. Mixing in inland and coastal waters, Academic Press, New York.

[5]Noutsopoulos G C, Yannopoulos P C. 1987. The round vertical turbulent buoyant jet, Journal of Hydraulic Research, 25(4):481 502

[6]Huai Wenxin, Fang Shenguang, Dai Huichao. 2006. Behavior of obstructed squared buoyant vertical jets in static ambient (I)-Verification of mathematical model and numerical method, Applied Mathematics and Mechanics, 27(5):645 652

[7]Huai Wenxin, Fang Shenguang. 2006. Rounded flowing states of obstructed buoyant jet, Applied Mathematics and Mechanics, 27(8):1133 1139

[8]Batchelor G K. 1970. An introduction to fluid dynamics, Cambridge University Press, Great Britain.

[9]Chen C J, Rodi W. 1976. A review of experimental data of vertical turbulent buoyant jets, Report No. 193, Iowa Inst. of Hydr. Res. 